\title{
Editorial
}

\section{Recent Advances in Musculoskeletal Tissue Regeneration}

\author{
Aliasger K. Salem ${ }^{1,2}$ \\ Received 16 May 2017; accepted 18 May 2017; published online 2 June 2017
}

There is a major need to promote regeneration of musculoskeletal tissues such as the bone and cartilage. Approaches to regenerating musculoskeletal tissues include the use of programmed stem cells, growth factors, gene therapies, and scaffold matrix materials. These approaches are being developed as standalone therapeutic strategies and in combination.

With respect to gene therapy applications, it is critical that clinically beneficial amounts of proteins are synthesized endogenously within and around the lesion in a sustained manner. By implanting gene-activated matrices (GAMs) in animal models, we have shown that sustained gene expression and continuous osteogenic protein production in situ can be achieved in a way that stimulates osteogenesis and bone repair within osseous defects $(1,2)$. We have also shown that critical parameters substantially affecting the therapeutic efficacy of gene therapy include the choice of osteogenic transgene(s), selection of vectors, the scaffold material, the wound environment, and the selection of delivery strategies (2-8). For example, we have developed a non-viral gene delivery system for bone regeneration utilizing a collagen scaffold to locally deliver complexes encoding for plateletderived growth factors (PDGF-B) (2). The in vivo regenerative capacity of the system was assessed in 5-mm diameter critical-sized calvarial defects in rats. In vivo studies showed significantly higher new bone volume/total volume in calvarial defects treated with GAMs following 4 weeks of implantation (14- and 44-fold higher) when compared to empty defects or empty scaffolds, respectively (2). Together, these findings suggest that non-viral gene-activated scaffolds are effective for bone regeneration and are an attractive therapeutic strategy with significant potential for clinical translation. Furthermore, simultaneous delivery of multiple genes is possible and customization is relatively straightforward. For

\footnotetext{
${ }^{1}$ University of Iowa, Iowa City, IA, USA.

${ }^{2}$ To whom correspondence should be addressed. (e-mail: aliasgersalem@uiowa.edu)
}

example, we have shown that co-delivering genes encoding fibroblast growth factor (FGF-2) and bone morphogenetic protein-2 (BMP-2) significantly enhances osteogenesis in human adipose-derived mesenchymal stem cells (hADMSC) over either gene alone (7). We also show that this combination of genes is effective at regenerating bone in a diaphyseal long bone radial defect diabetic rabbit model (9). More recently, we have been investigating the bone regenerative capacity of chemically modified ribonucleic acid (cmRNA). The osteogenic potential of BMSCs treated with cmRNA encoding for BMP-2 was validated by the enhanced expression of the bone-specific genes, osteocalcin and alkaline phosphatase as well as through the promotion of bone matrix deposition in vitro. In addition, using a calvarial bone defect model in rats, we have shown that cmRNA (encoding BMP2)-activated matrices promoted significantly enhanced bone regeneration compared to plasmid DNA (BMP-2)-activated matrices (1). In this theme issue, we report on the effectiveness of cmRNA encoding for BMP-2 versus cmRNA encoding for BMP-9 in rat calvarial defect models (10). We also provide a comprehensive review on development and testing of GAMs for bone regeneration (11).

This theme issue also covers recent advances in the areas of new materials for scaffolds and controlled release of agents that promote tissue regeneration. In the area of new materials for scaffolds for tissue regeneration, Elisseeff and colleagues show that a micronized porcine urinary bladder matrix can be used to reduce osteoarthritis induced by anterior cruciate ligament transection in mice (12). Berkland and colleagues show that colloidal gels composed of colloidal nanoparticles of hydroxyapatite, demineralized bone matrix, decellularized cartilage, and hyaluronic acid combined with bone morphogenetic protein-2 (BMP-2) and vascular endothelial growth factor generated significantly more bone in critical-sized rat calvarial defects than sham controls (13). In terms of evaluating the potential of controlled release of agents from scaffolds that can modulate and promote tissue repair, Livingston Arinzeh and colleagues show that controlled release of vanadium can stimulate mesenchymal stem cell osteochondrogenesis. This is evidenced by significant 
increases in alkaline phosphatase activity, osteocalcin production, and collagen synthesis when cells are cultured with vanadium-containing scaffolds versus empty scaffolds (14). In addition, Ulery and colleagues report on the controlled release of monobasic calcium phosphate-derived ions from polyester/ceramic composites to enhance osteoinductivity (15). Finally, Criswell and colleagues report on new data that casts doubt on the potential of growth differentiation factor 11 (GDF11). GDF11 was previously considered a strong circulating factor that could restore skeletal muscle function in aging animals (16). Criswell and colleagues show new data that adds to a growing body of evidence suggesting that GDF11 has negative effects including increased tissue fibrosis and impaired recovery of skeletal muscle function in older rats after injury $(17,18)$. Other recent AAPS J articles that are related to the topics presented in this theme issue include a report on the controlled release of simvastatin from in situ forming hydrogels to form bone (19), results describing a microsphere embedded wound dressing that provides controlled release of chitosan and sericin (20), a report on stem cell and minocycline-loaded hydrogels that inhibit growth of Staphylococcus aureus (21), a report on the controlled release of second-generation mTOR inhibitors to restrain inflammation in primary immune cells (22), and an article describing model selection and analysis of animal models of osteoarthritis (23).

\section{REFERENCES}

1. Elangovan S, et al. Chemically modified RNA activated matrices enhance bone regeneration. J Control Release. 2015;218:22-8.

2. Elangovan $\mathrm{S}$, et al. The enhancement of bone regeneration by gene activated matrix encoding for platelet derived growth factor. Biomaterials. 2014;35(2):737-47.

3. Atluri $\mathrm{K}$, et al. Gene-activated titanium surfaces promote in vitro osteogenesis. Int J Oral Maxillofac Implants. 2016.

4. D'Mello SR, et al. A pilot study evaluating combinatorial and simultaneous delivery of polyethylenimine-plasmid DNA complexes encoding for VEGF and PDGF for bone regeneration in calvarial bone defects. Curr Pharm Biotechnol. 2015;16(7):65560.

5. D'Mello S, Elangovan S, Salem AK. FGF2 gene activated matrices promote proliferation of bone marrow stromal cells. Arch Oral Biol. 2015;60(12):1742-9.

6. D'Mello S, et al. Incorporation of copper into chitosan scaffolds promotes bone regeneration in rat calvarial defects. Journal of
Biomedical Materials Research Part B-Applied Biomaterials. 2015;103(5):1044-9.

7. Atluri K, et al. Nanoplex-mediated codelivery of fibroblast growth factor and bone morphogenetic protein genes promotes osteogenesis in human adipocyte-derived mesenchymal stem cells. Mol Pharm. 2015;12(8):3032-42.

8. D'Mello S, et al. Characterization and evaluation of the efficacy of cationic complex mediated plasmid DNA delivery in human embryonic palatal mesenchyme cells. J Tissue Eng Regen Med, 2014; 10(11):927-937.

9. Khorsand B, et al. Regeneration of bone using nanoplex delivery of FGF-2 and BMP-2 genes in diaphyseal long bone radial defects in a diabetic rabbit model. J Control Release. 2017;248:53-9.

10. Khorsand $\mathrm{B}$, et al. A comparative study of the bone regenerative effect of chemically modified RNA encoding BMP-2 or BMP-9. AAPS J. 2017;19(2):438-46.

11. D'Mello S, et al. Bone regeneration using gene-activated matrices. AAPS J. 2017;19(1):43-53.

12. Jacobs HN, et al. Intra-articular injection of urinary bladder matrix reduces osteoarthritis development. AAPS J. 2017;19(1):141-9.

13. Townsend JM, et al. Colloidal gels with extracellular matrix particles and growth factors for bone regeneration in critical size rat calvarial defects. AAPS J. 2017;19(3):703-11.

14. Schussler SD, et al. Controlled release of vanadium from a composite scaffold stimulates mesenchymal stem cell osteochondrogenesis. AAPS J. 2017.

15. Ali Akbari Ghavimi S, et al. Controlled ion release from novel polyester/ceramic composites enhances osteoinductivity. AAPS J. 2017.

16. Sinha M, et al. Restoring systemic GDF11 levels reverses agerelated dysfunction in mouse skeletal muscle. Science. 2014;344(6184):649-52.

17. Zhou Y, et al. GDF11 treatment attenuates the recovery of skeletal muscle function after injury in older rats. AAPS J. 2017;19(2):431-7.

18. Hinken AC, et al. Lack of evidence for GDF11 as a rejuvenator of aged skeletal muscle satellite cells. Aging Cell. 2016;15(3):582-4.

19. Park YS, et al. Controlled release of simvastatin from in situ forming hydrogel triggers bone formation in MC3T3-E1 cells. AAPS J. 2013;15(2):367-76.

20. Aramwit P, Yamdech R, Ampawong S. Controlled release of chitosan and sericin from the microspheres-embedded wound dressing for the prolonged anti-microbial and wound healing efficacy. AAPS J. 2016;18(3):647-58.

21. Guerra AD, et al. Mesenchymal stromal/stem cell and minocycline-loaded hydrogels inhibit the growth of Staphylococcus aureus that evades immunomodulation of blood-derived leukocytes. AAPS J. 2015;17(3):620-30.

22. Gosselin EA, Tostanoski LH, Jewell CM. Controlled release of second generation mTOR inhibitors to restrain inflammation in primary immune cells. AAPS J. 2017.

23. Teeple E, et al. Animal models of osteoarthritis: challenges of model selection and analysis. AAPS J. 2013;15(2):438-46. 